\title{
Interconnection and synchronization of neuronal populations in the mouse medial septum/diagonal band of Broca
}

\author{
Richardson N. Leão, ${ }^{1,2}$ Zé H. Targino, ${ }^{2}$ Luis V. Colom, ${ }^{3 \dagger}$ and André Fisahn ${ }^{4}$ \\ ${ }^{1}$ The Beijer Laboratory for Gene and Neurosciences, Department of Neuroscience, Uppsala University, Uppsala, Sweden; \\ ${ }^{2}$ Brain Institute, Federal University of Rio Grande do Norte, Natal, Rio Grande do Norte, Brazil; ${ }^{3}$ Center for Biomedical \\ Studies, the University of Texas at Brownsville, Texas; and ${ }^{4}$ Neuronal Oscillations Laboratory, Division of Neurogeriatrics, \\ Center for Alzheimer Research, Department NVS, Karolinska Institutet, Stockholm, Sweden
}

Submitted 13 May 2014; accepted in final form 9 November 2014

Leão RN, Targino ZH, Colom LV, Fisahn A. Interconnection and synchronization of neuronal populations in the mouse medial septum/diagonal band of Broca. J Neurophysiol 113: 971-980, 2015. First published November 12, 2014; doi:10.1152/jn.00367.2014._ The medial septum/diagonal band of Broca (MS/DBB) is crucial for hippocampal theta rhythm generation $(4-12 \mathrm{~Hz})$. However, the mechanisms behind theta rhythmogenesis are still under debate. The MS/ DBB consists, in its majority, of three neuronal populations that use acetylcholine, GABA, or glutamate as neurotransmitter. While the firing patterns of septal neurons enable the MS/DBB to generate rhythmic output critical for the generation of the hippocampal theta rhythm, the ability to synchronize these action potentials is dependent on the interconnectivity between the three major MS/DBB neuronal populations, yet little is known about intraseptal connections. Here we assessed the connectivity between pairs of MS/DBB neurons with paired patch-clamp recordings. We found that glutamatergic and GABAergic neurons provide intraseptal connections and produce sizable currents in MS/DBB postsynaptic cells. We also analyzed linear and nonlinear relationships between the action potentials fired by pairs of neurons belonging to various MS/DBB neuronal populations. Our results show that while the synchrony index for action potential firing was significantly higher in pairs of GABAergic neurons, coherence of action potential firing in the theta range was similarly low in all pairs analyzed. Recurrence analysis demonstrated that individual action potentials were more recurrent in cholinergic neurons than in other cell types. Implementing sparse connectivity in a computer model of the MS/DBB network reproduced our experimental data. We conclude that the interplay between the intrinsic membrane properties of different MS/DBB neuronal populations and the connectivity among these populations underlie the ability of the MS/DBB network to critically contribute to hippocampal theta rhythmogenesis.

medial septum; theta rhythm; synchronization; cholinergic neuron; GABAergic neuron; glutamatergic neuron

THE CLASSICAL MODEL for the generation of theta oscillations postulates that nonrhythmic subcortical inputs are converted by the medial septum/diagonal band of Broca (MS/DBB) network into theta oscillation output, which, in turn, drives the hippocampus (Bland et al. 1999; reviewed in Buzsáki 2002). As such, the MS/DBB is critical for hippocampal theta rhythm generation (Vinogradova 1995; reviewed in Teles-Grilo Ruivo

\footnotetext{
† Deceased 27 March 2014

Address for reprint requests and other correspondence: A. Fisahn, Neuronal Oscillations Laboratory, Div. of Neurogeriatrics, Center for Alzheimer Research, Dept. NVS, Karolinska Institutet, Retzius väg 8, A3, SE-171 77 Stockholm, Sweden (e-mail: andre.fisahn@ki.se).
}

and Mellor 2013), a fact further supported by classical lesion studies (Andersen et al. 1979; Mitchell et al. 1982).

The MS/DBB is predominantly composed of cholinergic, GABAergic, and glutamatergic neuron populations (Colom et al. 2005; Sotty et al. 2003). A small proportion of neurons produce other neurotransmitters such as somatostatin and neuropeptide Y (NPY) (Gaspar et al. 1987). Classically, acetylcholine and GABA were considered the main neurotransmitters involved in theta generation, but recent studies suggest that glutamate (Huh et al. 2010), the most abundant excitatory neurotransmitter in the CNS, is also essential for theta oscillations. To date, it is not known how the cholinergic, GABAergic, and glutamatergic neuronal populations are interconnected and how specific connection properties govern action potential (AP) synchronization and contribute to the generation of rhythmic activity.

To remediate this paucity of evidence, we decided to investigate MS/DBB neuronal connectivity by using septal slices from mouse brains. MS/DBB neurons receive abundant excitatory glutamatergic inputs from hypothalamic nuclei that are severed in the slice preparation (Kiss et al. 2000). This input loss may contribute to the in vitro MS/DBB neuronal network's reduced rhythmogenic properties. Thus we decided to compensate for this excitatory deficit by bath-applying kainate to the slice preparation to improve the rhythmogenic capabilities of the MS/DBB neuronal network (Garner et al. 2005).

Using paired patch-clamp recordings, we found that septal GABAergic and glutamatergic neurons form synapses within the MS/DBB. Furthermore, we established that under in vitro conditions, despite the moderate firing rhythmicity in all neuronal populations, the synchrony index for AP firing is significantly higher in pairs of GABAergic neurons compared with all other pairs of neurons. Conversely, only cholinergic neurons fired APs in the theta frequency range and showed recurrence as revealed by recurrence analysis, but coherence in theta was low in all pairs studied. We suggest that the interplay between intrinsic membrane properties of the different MS/ DBB cell classes with the sparse connectivity is insufficient for in vitro $\mathrm{MS} / \mathrm{DBB}$ neuronal network to generate the theta rhythm.

\section{MATERIALS AND METHODS}

Animals. Experiments were approved by Norra Stockholms Djurförsöksetiska Nämnd (ethical permit N45/13) and were carried out in accordance with the European Community Council Directive of 24 November 1986 (86/609/EEC). Mice were deeply anesthetized 
with isoflurane before being killed by decapitation. Coronal MS/DBB slices of P17-21 mice of either sex were made from the following strains: C57/B6, BAC-vesicular glutamate transporter 2 (VGLUT2):: Cre (Vglut2-Cre) (Borgius et al. 2010), glutamic acid decarboxylase 2

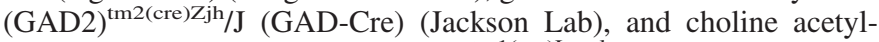
transferase (ChAT) B6;129S6-ChAT ${ }^{\mathrm{tm} 1 \text { (cre)Lowl } / J}$ (ChAT-Cre) (Jackson Lab). Vglut2-Cre mice were crossed with Z/EG reporter mice (Borgius et al. 2010; Leao et al. 2012), while GAD-Cre and ChAT-Cre mice were crossed with B6;129S6-Gt(ROSA)26Sor ${ }^{\text {tm9(CAG-tdTomato)Hze/J (Jackson }}$ Lab). MS/DBB slicing followed previously described procedures (Leao et al. 2012).

Electrophysiology. Coronal MS/DBB slices $(300 \mu \mathrm{m})$ were cut in high-sucrose artificial cerebrospinal fluid (ACSF, in mM: $2.49 \mathrm{KCl}$, $1.43 \mathrm{NaH}_{2} \mathrm{PO}_{4}, 26 \mathrm{NaHCO}_{3}, 10$ glucose, 252 sucrose, $1 \mathrm{CaCl}_{2}, 4$ $\mathrm{MgCl}_{2}$ ) and immediately transferred to a submerged holding chamber containing ACSF (in mM: $124 \mathrm{NaCl}, 3.5 \mathrm{KCl}, 1.25 \mathrm{NaH}_{2} \mathrm{PO}_{4}, 1.5$ $\mathrm{MgCl}_{2}, 1.5 \mathrm{CaCl}_{2}, 30 \mathrm{NaHCO}_{3}, 10$ glucose) constantly bubbled with $95 \% \mathrm{O}_{2}-5 \% \mathrm{CO}_{2}$. For recordings, slices were transferred to a submerged recording chamber $\left(30^{\circ} \mathrm{C}\right)$ under an upright microscope equipped with infrared and DIC optics and perfused with oxygenated ACSF at a rate of $\sim 1 \mathrm{ml} / \mathrm{min}$ (composition as holding chamber ACSF given above). Neurons were identified by green or red fluorescence after blue (eGFP) or green (tdTomato) excitation (Leao et al. 2012). In some experiments we used $50 \mu \mathrm{M}$ D-AP5 (Sigma) to block NMDA receptors, $10 \mu \mathrm{M}$ CNQX (Sigma) to block AMPA/kainate receptors, $10 \mu \mathrm{M}$ picrotoxin (PTX; Sigma) to block $\mathrm{GABA}_{\mathrm{A}}$ receptors, and/or $50 \mu \mathrm{M}$ mecamylamine hydrochloride (MEC; Tocris) to block nicotinic cholinergic receptors. Internal recording solutions were based on ACSF for cell-attached recordings, on K-gluconate for current-clamp recordings (in mM: $17.5 \mathrm{KCl}, 122.5 \mathrm{~K}$-gluconate, $9 \mathrm{NaCl}, 1 \mathrm{MgCl}_{2}$, $3 \mathrm{Mg}$-ATP, 0.3 GTP-Tris, 1 HEPES, 0.2 EGTA; pH adjusted to 7.2 with $\mathrm{KOH}$ ), or on $\mathrm{CsCl}$ for voltage-clamp recordings (in mM: 120 $\mathrm{CsCl}, 4 \mathrm{NaCl}, 4 \mathrm{MgCl}_{2}, 0.001 \mathrm{CaCl}_{2}, 10 \mathrm{HEPES}, 3 \mathrm{Mg}$-ATP, 0.3 GTP-Tris, and 10 EGTA). Recording electrodes were produced from borosilicate glass and had a resistance between 3 and $5 \mathrm{M} \Omega$ (voltage clamp) or between 5 and $9 \mathrm{M} \Omega$ (current clamp and cell attached). Data were recorded with a Multiclamp 700B (Molecular Devices) amplifier or a Dagan BVC-700 (Dagan) and an Axopatch 200B amplifier (Molecular Devices) and digitized with a DAQ card (National Instruments) and the software WinWCP and WinEDR (Dr. John Dempster, http://spider.science.strath.ac.uk/sipbs/software_ses.htm).

Data analysis. All data analysis was performed with custom programs written in MATLAB using the Statistics and Signal Processing toolboxes (MathWorks). Spike detection was performed with a threshold algorithm. A vector $X$ containing spike times was computed by making $X_{n}=1$ with $n$ equal to the time of a given spike with 1-ms resolution while the remaining values were equal to 0 (Hilscher et al. 2013). In the time domain, the degree of synchronization was estimated by a "synchronization index" (SI) defined by the peak of the normalized cross-correlation value (Hilscher et al. 2013). Only peaks at positive time bins (lags) were used in the SI calculation. Crosscorrelations were calculated with the MATLAB xcorr command with the option coeff that normalizes the cross-correlogram by normalizing autocorrelations at zero lag to 1 (Hilscher et al. 2013). To determine the relationship between the AP firing of two neurons in the frequency domain, coherence between spike trains was calculated with the MATLAB command "mscohere." Confidence intervals for crosscorrelation and coherence for single pairs were obtained by randomizing interevent intervals (Hung et al. 2002). Recurrence quantification analysis (RQA) (Marwan et al. 2002; Zbilut and Webber 1992) was performed in series containing spike times as previously reported (Leao et al. 2005) using the Cross Recurrence Plot Toolbox (http:// tocsy.pik-potsdam.de/CRPtoolbox/) (Marwan et al. 2002).

Single-cell reverse transcriptase PCR. After cell-attached or whole cell recordings, the cytoplasm and organelles of the cells were sucked into the recording pipette tip as previously described (Leao et al. 2012). Buffers and cDNA conversion are described in Leao et al.
(2012). However, in this work, we used two-round PCR (nested PCR; Birgner et al. 2010) to detect ChAT, GAD, or Vglut2 cDNA. For the first round of PCR we use the following mix: $1.5 \mathrm{mM} \mathrm{MgCl}_{2}, 10 \mathrm{pmol}$ of each primer, $1.0 \mathrm{U}$ of platinum Taq-DNA polymerase (Invitrogen),

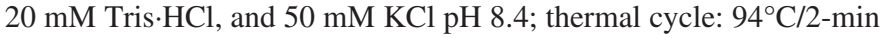
denaturation step followed by 35 cycles of $94^{\circ} \mathrm{C} / 50 \mathrm{~s}, 55^{\circ} \mathrm{C} / 45 \mathrm{~s}$, and $72^{\circ} \mathrm{C} / 45 \mathrm{~s}$. The same mix was used for the second nested PCR, but instead of mixing the original template we added $10 \%$ of the first PCR reaction as template. Second-round PCR products were visualized on $2 \%$ agarose gels. Primers were designed based upon sequences deposited in the GenBank database (www.ncbi.nlm.nih.gov/nucleotide) and include the following: for the first round: ChAT forward: CAGCTCTGCTACTCTGGATTAAGAA, reverse: CACCAGTGTGTCCTGGGTATG; GAD1 forward: CCAATAGCCTGGAAGAGAAGAG, reverse: TCCCATCACCATCTTTATTTGA; Vglut2 forward: GCCGCTACATCATAGCCATC, reverse: GCTCTCTCCAATGCTCTCCTC and for the second round: ChAT (product size: $311 \mathrm{bp}$ ) forward: GCCACCTACCTTCAGTGCAT, reverse: GCCTTGTAGCTAAGCACACCA; GAD (product size: 404 bp) forward: GTCCTCCAAGAACCTGCTTTC, reverse: TCAGCCATTCACCAGCTAAAC; Vglut2 (product size: 506 bp) forward: ACATGGTCAACAACAGCACTATC, reverse: ATAAGACACCAGAAGCCAGAACA.

MS/DBB computer model. We simulated groups of MS/DBB neurons with the Brian Simulator (Goodman and Brette 2008) using a 64-core computer cluster with 192 GB RAM. The network architecture was similar to a previously described model (Leao et al. 2012; Wang 2002), but we added a population of spontaneously firing cholinergic neurons. The network consisted of 50 cholinergic, 100 GABAergic, and 100 glutamatergic cells (Bañuelos et al. 2013; Colom et al. 2005). Equations for GABAergic and glutamatergic neurons (using the Hodgkin and Huxley formalism) were described in Leao et al. (2012), and cholinergic neurons were modeled according to the following equations:

$$
\begin{aligned}
& \mathrm{d} V / \mathrm{d} t=\left(\begin{array}{c}
-g_{1}\left(V-V_{1}\right)+g_{\mathrm{e}}\left(V_{\mathrm{e}}-V\right)+g_{\mathrm{i}}\left(V_{\mathrm{i}}-V\right)-I_{\mathrm{Ca}} \cdots \\
-I_{\mathrm{KC}}-I_{\mathrm{K}_{\mathrm{AHP}}}-I_{\mathrm{M}}-I_{\mathrm{K}_{\mathrm{P}}}-I_{\mathrm{Na}_{\mathrm{P}}}-I_{\mathrm{h}}-I_{\mathrm{Na}}-I_{\mathrm{K}}
\end{array}\right) / C \\
& I_{\mathrm{Ca}}=\overline{g_{\mathrm{Ca}}} s^{2}\left(V-V_{\mathrm{Ca}}\right) \\
& I_{\mathrm{KC}}=\overline{g_{\mathrm{KC}}} \cdot C a \cdot\left(V-V_{\mathrm{K}}\right) \text {, with } 0<C a<1 \\
& I_{\mathrm{K}_{\mathrm{AHP}}}=\overline{g_{\mathrm{K}_{\mathrm{AHP}}}} \cdot q \cdot\left(V-V_{\mathrm{K}}\right) \\
& I_{\mathrm{M}}=\overline{g_{\mathrm{M}}} \cdot r \cdot\left(V-V_{\mathrm{K}}\right) \\
& I_{\mathrm{K}_{\mathrm{P}}}=\overline{g_{\mathrm{K}_{\mathrm{P}}}} \cdot a \cdot\left(V-V_{\mathrm{K}}\right) \\
& I_{\mathrm{Na}_{\mathrm{p}}}=\overline{g_{\mathrm{Na}_{\mathrm{p}}}} \cdot b \cdot\left(V-V_{\mathrm{Na}}\right) \\
& I_{\mathrm{h}}=\overline{g_{\mathrm{h}}} \cdot u \cdot\left(V-V_{\mathrm{h}}\right) \\
& I_{\mathrm{Na}}=\overline{g_{\mathrm{Na}}} m^{3} h\left(V-V_{\mathrm{Na}}\right) \\
& I_{\mathrm{K}}=\overline{g_{\mathrm{K}}} \cdot n^{4} \cdot\left(V-V_{\mathrm{K}}\right) \\
& \mathrm{d} C a / \mathrm{d} t=-1,300 I_{\mathrm{Ca}}-0.075 C a \\
& \mathrm{~d} g_{\mathrm{e}} / \mathrm{d} t=-g_{\mathrm{e}}\left(1 / \tau_{\mathrm{e}}\right) \\
& \mathrm{d} g_{\mathrm{i}} / \mathrm{d} t=-g_{\mathrm{i}}\left(1 / \tau_{\mathrm{i}}\right)
\end{aligned}
$$

Evolution variables $r, a, b$, and $u$ were calculated by

$$
\mathrm{d} x / \mathrm{d} t=\left(x_{\infty}-x\right) / \tau_{x}, \text { where } x=r, a, b, \text { or } u
$$

Evolution variables $q, s, m, n$ and $h$ were calculated with the classical Hodgkin and Huxley connotation (Hodgkin and Huxley 1952):

$$
\mathrm{d} x / \mathrm{d} t=\alpha_{x}(1-x)-\beta_{x} x, \text { where } x=q, s, m, n, \text { or } h
$$

where

$$
\begin{gathered}
\tau_{r}=4135.7 / 164.64 e^{0.12(V-0.05)}+0.33 e^{-0.1(V-0.05)}+35.66 \\
r_{\infty}=\left(1+e^{-0.1(V+2253)}\right)^{-1}
\end{gathered}
$$




$$
\begin{gathered}
\tau_{a}=30 \\
a_{\infty}=\left(1+e^{-0.1(V+63)}\right)^{-1} \\
\tau_{b}=0.05 \\
b_{\infty}=\left(1+e^{-0.2(V+60)}\right)^{-1} \\
\tau_{u}=10,000 /\left(237 e^{(V+50) / 12}+17 e^{-(V+50) 25}\right)^{+6} \\
u_{\infty}=\left(1+e^{(V+76) / 7}\right)^{-1} \\
\tau_{c}=\left(2 e^{(V+53.5) / 27}\right)^{-1} \\
c_{\infty}=\left(1+e^{-(V+15)}\right)^{-1} \\
\alpha_{s}=1.6 /\left(1+e^{-0.072(V-5)}\right) \\
\beta_{s}=0.2(V+8.9) /\left(e^{(V+8.9) / 5}-1\right) \\
\alpha_{q}=0.01 C a / 500 \\
\beta_{q}=1 / \tau_{\mathrm{K}_{\mathrm{AHP}}} \\
\alpha_{m}=0.32(13-V+v) /\left(e^{(13-V+v) / 4}-1\right) \\
\beta_{m}=0.28(V-40+v) /\left(e^{(V-40+v) / 5}-1\right) \\
\alpha_{h}=0.128 e^{(17-V+v) / 18} \\
\beta_{h}=4 / 1+e^{(40-V+v) / 5} \\
\beta_{n}=0.5 e^{(10-V+v) / 40} \\
\alpha_{n}=0.032(15-V+v) /\left(e^{(15-V+v) / 5}-1\right) \\
\end{gathered}
$$

with

$$
\begin{aligned}
V_{1} & =-58 \mathrm{mV} \\
g_{1} & =0.1 \mathrm{nS} \\
\overline{g_{\mathrm{m}}} & =0.2 \mathrm{nS} \\
\overline{g_{\mathrm{Ca}}} & =10 \mathrm{nS} \\
\overline{g_{\mathrm{K}}} & =10 \mathrm{nS} \\
\overline{g_{\mathrm{K}}} & =1 \mathrm{nS} \\
\overline{g_{\mathrm{KP}}} & =0.025 \mathrm{nS} \\
\overline{g_{\mathrm{h}}} & =1.2 \mathrm{nS} \\
\overline{g_{\mathrm{Na}}} & =45 \mathrm{nS} \\
\overline{g_{\mathrm{NaP}}} & =30 \mathrm{nS} \\
\overline{g_{\mathrm{K}}} & =10 \mathrm{nS} \\
v & =-63 \mathrm{mV} \\
\tau_{\mathrm{K}} & =1,000 \mathrm{~ms}
\end{aligned}
$$

\section{RESULTS}

We performed paired patch-clamp recordings of 183 neuron pairs in whole cell mode and of 50 pairs in cell-attached mode to investigate how the three major neuronal populations of the $\mathrm{MS} / \mathrm{DBB}$ are interconnected and whether they interact with each other to produce synchronous activity in vitro. Three different transgenic animal lines were used to identify the neuronal class based on neurotransmitter production (acetylcholine, GABA, or glutamate) of one or both neurons in the recorded pairs (ChAT-Cre, GAD-Cre, and Vglut2-Cre) crossed with either eGFP or tdTomato reporter mice (Fig. $1, A-C$ ). No difference in cell distribution was found when comparing Cre lines crossed with either reporter line. The identity of the unlabeled neuron in the pair was determined by single-cell reverse transcriptase PCR (Fig. 1D). No difference in firing frequency was found between a cell identified with the Cre/lox system or single-cell PCR; hence, all data for a single neuronal class were pooled together.

We first used paired current-/voltage-clamp recordings to assess whether pairs of different MS/DBB neuron classes are monosynaptically connected. We limited the distance between pairs from 0 to $0.2 \mathrm{~mm}$; hence it was not possible to determine topographic density of intraseptal connections. In these experiments, the nature of the presynaptic cell was determined by expression of fluorescent proteins while the postsynaptic cell type was either determined by single-cell PCR or, when belonging to the same neuronal class as the presynaptic neuron, by expression of fluorescent proteins. To increase the probability of finding connected neurons, we followed the axon of the presynaptic fluorescent neuron and patched postsynaptic cells' apposing varicosities (Fig. 2A). The proportion of connected pairs formed by a combination of different cell types is shown in Table 1. We found no ionotropic (nicotinic) synapses between cell pairs where the presynaptic neuron was ChAT+ and the postsynaptic neuron belonged to any of the three major MS/DBB neuronal populations. Glutamatergic synapses (Vglut2+ presynaptic neurons from Vglut2-Cre mice) were isolated by perfusing PTX and MEC onto MS/DBB slices. These synapses exhibited postsynaptic currents with mean half-width of $2.39 \pm 0.11 \mathrm{~ms}$ and mean amplitude of $19.48 \pm$ $0.50 \mathrm{pA}$ and delay of $5.11 \pm 0.98 \mathrm{~ms}(n=10$ pairs; Fig. $2, B$ and $C$ ). Distributions of half-width and amplitude of 350 excitatory postsynaptic currents (EPSCs) from all pairs with a Vglut2+ presynaptic cell are shown in Fig. 2C. GABAergic synapses (GAD + presynaptic neurons from GAD-Cre mice) exhibited postsynaptic currents with mean half-width of $3.16 \pm$ $0.10 \mathrm{~ms}$, amplitude of $40.42 \pm 1.39 \mathrm{pA}$, and delay of $4.32 \pm$ $0.42 \mathrm{~ms}$ ( $n=13$ pairs; Fig. 2, $B$ and $D$ ). Distributions of half-widths and amplitudes of 350 inhibitory postsynaptic currents (IPSCs) from all pairs with a GAD + presynaptic cell are shown in Fig. $2 D$.

We next assessed whether MS/DBB neuron pairs fire synchronously in both time (cross-correlation) and frequency (coherence) domains in response to the application of $100 \mathrm{nM}$ kainate. Recordings were performed 10 min after the kainate perfusion onset. Pairs of cholinergic neurons fired APs registered in cell-attached configuration with a SI of $0.18 \pm 0.05$ ( $n=12$ pairs, Fig. $3, A$ and $D$ ). GAD/ChAT cell pairs fired APs with a SI of $0.10 \pm 0.05(n=8$ pairs; Fig. $3, B$ and $D)$, and GAD/GAD cell pairs fired APs with a SI of $0.40 \pm 0.11$ for $(n=9$ pairs; Fig. $3, C$ and $D$ ). The SI (see MATERIALS AND METHODS and Hilscher et al. 2013 for definition) of GAD/GAD cell pairs was significantly higher than that for ChAT/ChAT pairs $(P=0.04, t$-test $)$ and ChAT/GAD pairs $(P=0.04, t$-test; Fig. 3D). As observed previously, the large majority of Vglut2+ neurons fire APs at very low frequency even in the presence of $100 \mathrm{nM}$ kainate (Leao et al. 2012), and hence we were able to calculate the SI only in 4 of $13 \mathrm{Vglut} 2 / \mathrm{GAD}$ neuron pairs (equal to $1.6 \pm 0.4$ ) and 4 of 17 VGlut2/ChAT pairs (equal to $0.8 \pm 0.3$ ). Both of these SI values were not significantly different from the SI of GAD/GAD pairs. It is important to note that the highest correlation coefficients (SI) of the firing of GAD/GAD neuron pairs occurred near zero lag (Fig. 3D).

The level of phase-locking in the frequency domain was accessed by computing coherence spectra. Because of the classical association of the MS/DBB with theta pacemaking, 
Fig. 1. Fluorescence images of medial septum/diagonal band of Broca (MS/DBB) of ChAT-Cre, GAD-Cre, and Vglut2-Cre lines crossed with Tomato or eGFP reporter lines. $A-C$ : Cre lines used to differentiate cell types in the MS/DBB. The MS/DBB region is delineated by the dashed yellow lines. Scale bar, $200 \mu \mathrm{m}$. D: example of a gel obtained from single-cell RT-PCR showing a VGlut2+ cell.
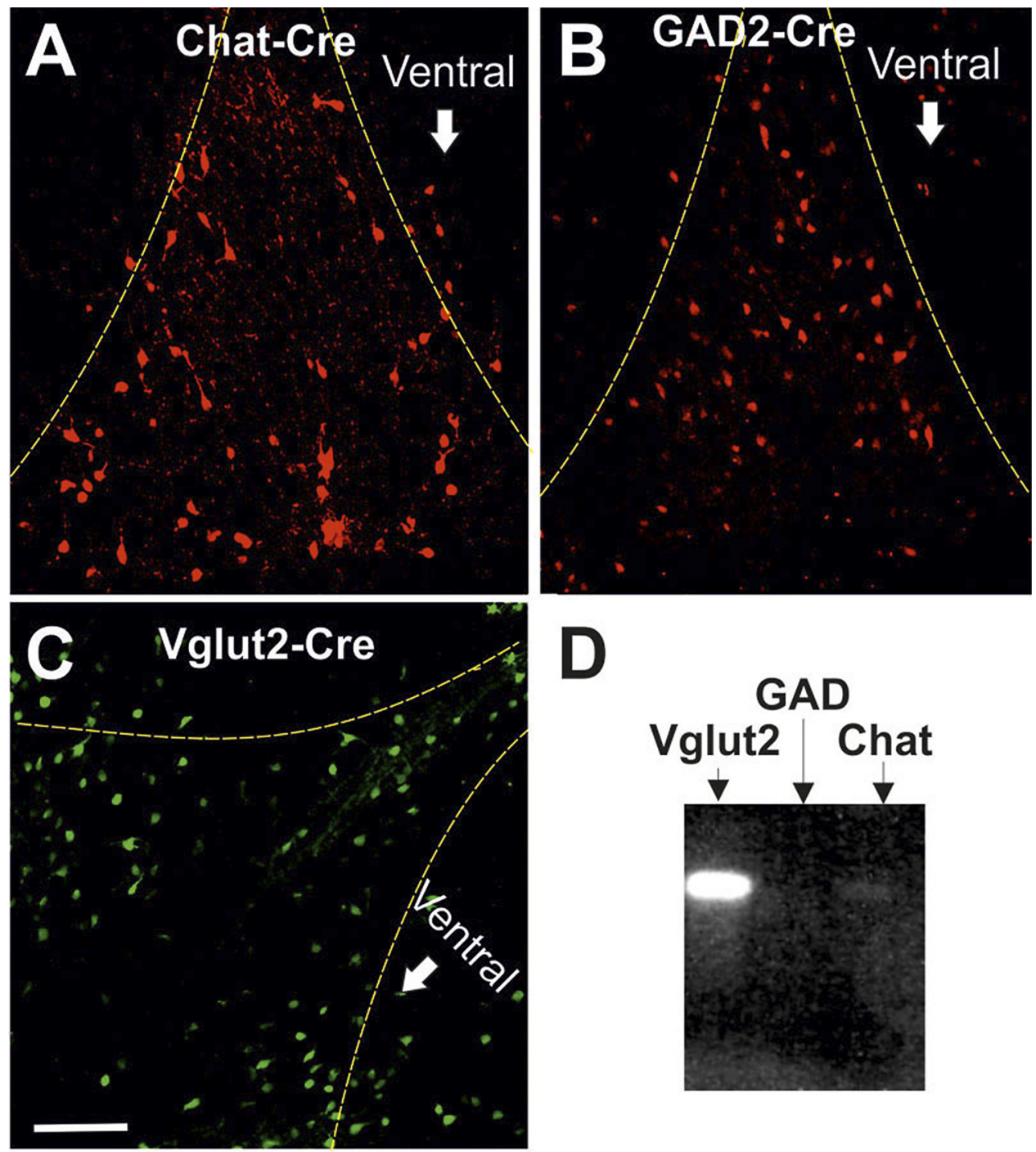

we restricted the coherence analysis to the theta frequency range $(4-12 \mathrm{~Hz})$. We found no significant differences in coherence between different pairs (Fig. 4). We also analyzed the power spectral density (PSD) of individual neurons: ChAT + neurons showed a prominent peak between 3 and $6 \mathrm{~Hz}$ $\left[4.1 \pm 0.6 \times 10^{-5}\right.$ arbitrary units (a.u.), $n=36$; Fig. $4, A$ and $B]$ compared with GAD + neurons $\left(0.5 \pm 0.1 \times 10^{-5}\right.$ a.u., $n=$ $34, P=1.8 \times 10^{-7}$, Fig. $4, B$ and $C$ ) or Vglut $2+$ neurons $\left(0.2 \pm 0.1 \times 10^{-5}\right.$ a.u., $\left.n=8, P=0.02\right)$.

The AP firing rhythmicity of the different neurons in the MS/DBB was further investigated with RQA (Leão et al. 2005; Marwan et al. 2002). While cross-correlation or spectral coherence may define linear associations between signals, these measurements are insensitive to nonlinear relationships. RQA is a method based on recurrence plots for nonlinear analysis of dynamical systems (Marwan et al. 2002). We applied RQA for spike data of individual cells with spiking frequency $>2 \mathrm{~Hz}$ (to provide sufficient data points for RQA calculations; Leão et al. 2005). A total of $21 \mathrm{ChAT}+$ and $25 \mathrm{GAD}+$ neurons (no Vglut2 + cells) satisfied the firing frequency criteria. The rate of recurrence (\% recurrence) on the recurrence plot is a simple measurement in RQA, and its definition corresponds to the definition of correlation sum and estimates the chance of a given state to (re)occur (Marwan et al. 2002). GAD+ neuron firing produced a mean recurrence of $5.1 \pm 1.1 \%$, whereas ChAT + neuron firing produced a mean recurrence of $20.2 \pm$ $3.2 \%\left(P=8 \times 10^{-6}, t\right.$-test; Fig. 5). A further measurement in RQA is the proportion of recurrent points in a recurrence plot, which quantifies repeating patterns in the signal (Zbilut and Webber 1992). This determinism for spike data from GAD+ neurons was equal to $75.3 \pm 2.1 \%$, whereas the same measure for ChAT+ neurons was equal to $88.4 \pm 1.9 \%\left(P=8 \times 10^{-6}\right.$, $t$-test; Fig. 5). These results confirm that ChAT + neurons show a higher degree of rhythmicity than their GAD + counterparts.

Finally, we implemented a mathematical model of the MS/ DBB neuronal network similar to a previously described architecture (Leao et al. 2012) but with the addition of cholinergic neurons. The model consisted of 50 cholinergic, 100 glutamatergic, and 100 GABAergic neurons (Fig. 6A). We varied conductances for $I_{\mathrm{M}}, I_{\mathrm{h}}, I_{\mathrm{AHP}}$, and persistent sodium and potassium currents $\pm 10 \%$ in all cell types to add inhomogeneity to the network. Excitation to all model cells was provided by 100 "generic" neurons firing APs with Poisson statistics (Goodman and Brette 2008). In control conditions (neurons interconnected as in Table 1), cholinergic neurons showed regular firing at $\sim 3 \mathrm{~Hz}$ (Fig. $6, B$ and $C$ ). It is important to 
A

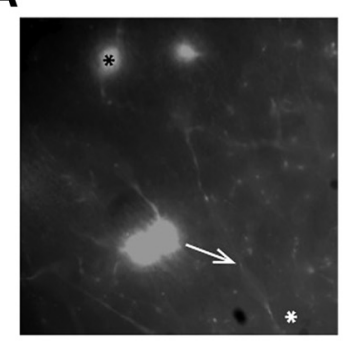

B

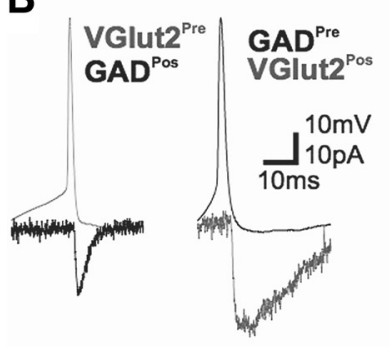

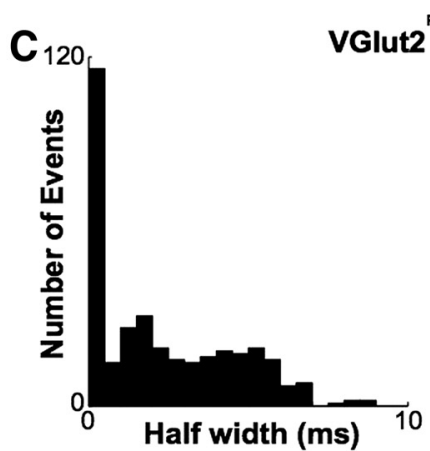
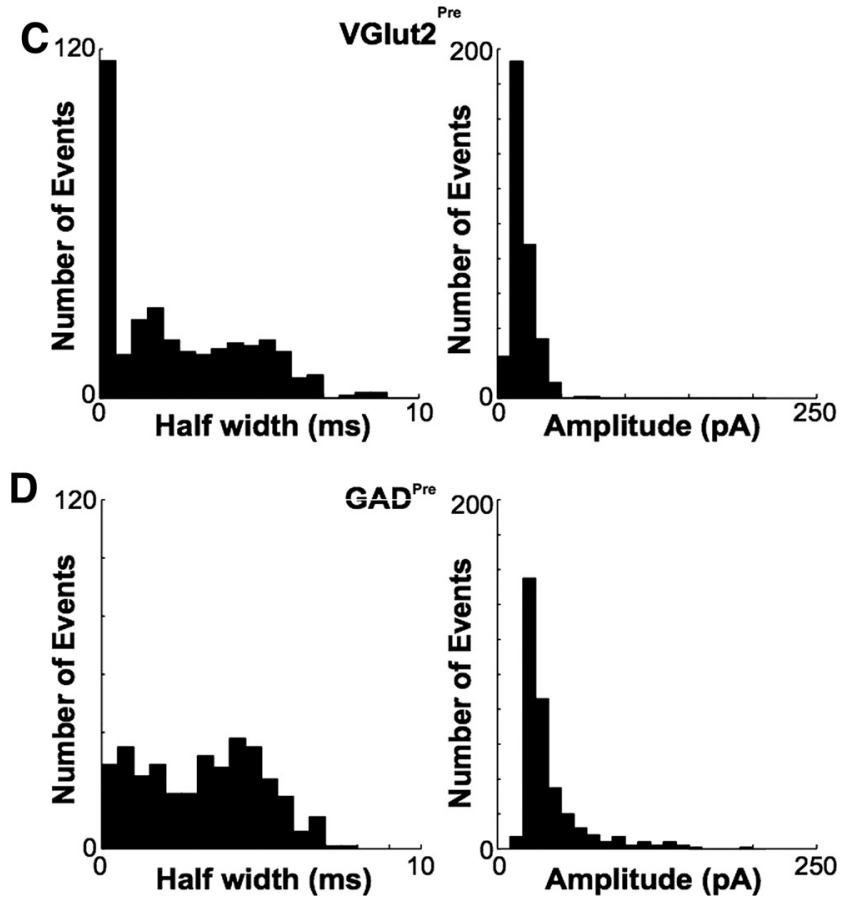

Fig. 2. Presynaptic spikes produce large excitatory (EPSCs) and inhibitory (IPSCs) postsynaptic currents and in pairs of MS/DBB neurons. $A$ : example of a paired patch-clamp recording. In this example the fluorescent presynaptic neuron is GAD2 + (black asterisk) and the postsynaptic neuron (white asterisk) was targeted after the visualization of an axonal varicosity apposing the postsynaptic cell dendrite (white arrow). $B$, left: example traces of a paired patch-clamp recording of a Vglut2+ neuron connected to a GAD2+ neuron. The black trace represents an EPSC recorded in the postsynaptic cell $(\mathrm{GAD} 2+)$ in response to a presynaptic spike of a VGlut $2+$ neuron. Right: example traces of a paired patch-clamp recording of a GAD2+ neuron connected to a VGlut2+ neuron. The black trace represents an IPSC recorded in the postsynaptic cell (VGlut2+) in response to a presynaptic spike of a GAD2 + neuron. The IPSC is shown inverted (as an inward current) for clarity. $C$, left: EPSC half-width histogram. Right: histogram of EPSC amplitudes elicited by a single action potential (AP) in VGlut2+ cells. D, left: IPSC half-width histogram. Right: histogram of IPSC amplitudes elicited by a single AP in GAD2+ presynaptic cells. stress that there were no feedback connections from cholinergic neurons in this model. Most glutamatergic neurons fired sparsely (Leao et al. 2012), except from small clusters of cells that fired irregularly at higher frequencies (Fig. 6, $B$ and $C$ ). GABAergic neurons fired spikes irregularly at relatively high frequencies (Fig. 6, $B$ and $C$ ). Increasing the number of GABAergic or glutamatergic synapses or GABAergic synapses did not generate rhythmicity in GABAergic or glutamatergic neurons and had little effect on cholinergic neuron firing regularity. Figure $6 C$ shows normalized PSDs (PSD divided by its PSD integral) of all cell types in control conditions and when the numbers of glutamatergic and GABAergic synapses were increased to 2 or 4 times the control values (Table 2). Increasing GABAergic or glutamatergic synaptic strength had no effect on cell rhythmicity (data not shown). On the other hand, increasing the excitability of glutamatergic neurons by decreasing $I_{\mathrm{M}}$ in these neurons $\left(g_{\mathrm{M}}=0.7 \mathrm{nS}\right)$ generated regular spiking rates (around $35 \mathrm{~Hz}$ ) in both glutamatergic and GABAergic neurons while causing a decrease in the $3-\mathrm{Hz}$ peak power of cholinergic neuron firing. These data indicate that phasic glutamatergic/GABAergic excitation/inhibition will not generate per se regular firing rates at theta frequencies.

Next, we assessed whether synchronization within the model network cells is affected by changes in synaptic strength or

Table 1. Connectivity among different MS/DBB cell classes

\begin{tabular}{ccr}
\hline \hline Presynaptic Cell & Postsynaptic Cell & Connections \\
\hline GAD & Vglut2 & $11.4 \%(4 / 35)$ \\
GAD & GAD & $14.0 \%(7 / 50)$ \\
Vglut2 & GAD & $10.0 \%(5 / 39)$ \\
Vglut2 & Vglut2 & $5.3 \%(4 / 29)$ \\
Vglut2 & ChAT & $8.3 \%(1 / 12)$ \\
GAD & ChAT & $11.1 \%(2 / 18)$ \\
\hline
\end{tabular}

MS/DBB, medial septum/diagonal band of Broca; GAD, glutamic acid decarboxylase; Vglut2, vesicular glutamate transporter 2; ChAT, choline acetyltransferase. connectivity. Synaptic strength had little effect on mean spiking SI between neurons of the same or different classes (glutamatergic $\times$ glutamatergic, glutamatergic $\times$ GABAergic, glutamatergic $\times$ cholinergic, GABAergic $\times$ cholinergic, and cholinergic $\times$ cholinergic). However, increasing connectivity, especially glutamatergic, dramatically increased SI between glutamatergic neuron pairs, between GABAergic neuron pairs, and between GABAergic and glutamatergic neurons (Table 2, Fig. 7). The largest increase in SI was observed between glutamatergic and GABAergic cells when the number of glutamatergic connections in the model was quadrupled (Table 2, Fig. 7). No changes in SI were observed between pairs that included cholinergic neurons. These results suggest that rather than generating regular firing at theta frequencies, intraseptal glutamatergic and GABAergic connections may synchronize neurons within the MS/DBB network.

\section{DISCUSSION}

Paired patch-clamp recordings were used to analyze GABAergic and glutamatergic synaptic currents in MS/DBB neuron pairs. MS/DBB major neuronal populations were identified by the expression of fluorescent proteins in GABAergic, cholinergic, and glutamatergic neurons or post hoc by singlecell reverse transcriptase PCR. To increase the likelihood of finding connected cells, we tracked fluorescent axons from presynaptic cells and targeted postsynaptic neurons that appeared (visually) to be connected to these axons. Hence, we cannot infer the "real" connectivity of the MS/DBB network. Nevertheless, we found that MS/DBB GABAergic and glutamatergic presynaptic neurons produce sizable postsynaptic currents in other MS/DBB neurons, evincing the existence of a functional intraseptal network. Paired cell-attached recordings of pairs of MS/DBB cells suggest a diverse degree of synchronization among different neuron populations in vitro. To increase our insight into the intrinsic mechanisms of theta rhythmogenesis in MS/DBB networks, we implemented a Hodgkin 

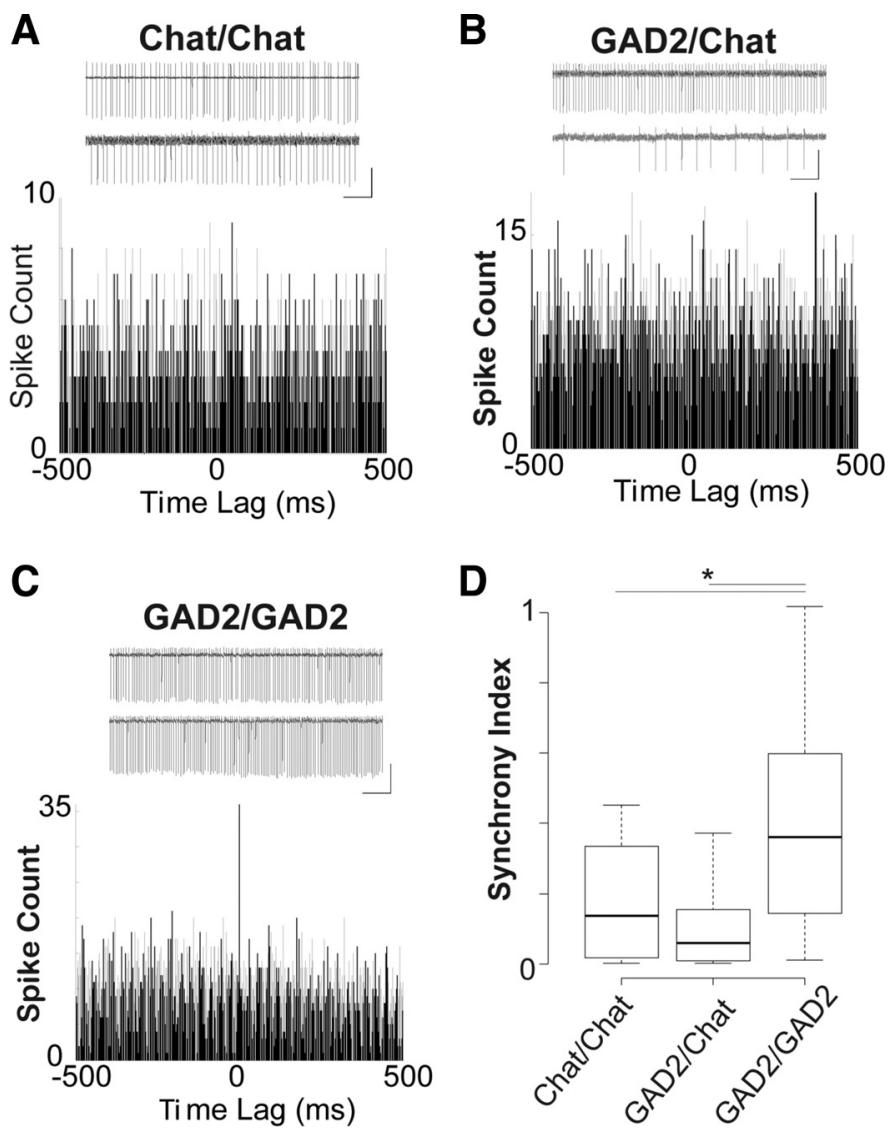

Fig. 3. Spikes of GABAergic neuron pairs are more synchronized than in ChAT/ChAT or GAD2/ChAT pairs. A: examples of cell-attached recordings of a pair of ChAT neurons and their respective cross-correlogram. Scale bar, $2 \mathrm{~s} / 1$ $\mathrm{mV}$. B: same as $A$ for a pair composed of a GAD2 (top) and a ChAT (bottom) cell. $C$ : same as $A$ for a pair of GAD2 cells. Scale bar, $2 \mathrm{~s} / 1 \mathrm{mV}$. $D$ : box plots showing mean synchrony index of ChAT/ChAT, GAD2/ChAT, and GAD2/ GAD2 pairs $(* P<0.05, t$-test $)$.

and Huxley-like 250-neuron MS-DBB network model using synaptic data produced in this study and intrinsic membrane properties of various MS/DBB neurons found elsewhere (Henderson et al. 2010; Leao et al. 2012; Morris et al. 2004). Our simulations showed that in a network composed purely of glutamatergic or GABAergic synapses, changes in synaptic strength or connectivity have some effect on synchrony but little influence on theta rhythmicity. On the other hand, postsynaptic currents produced by slow voltage-dependent ion channels had a large impact in firing at theta frequencies.

In this work, we show that GABAergic and glutamatergic MS/DBB neurons connect to other neurons within the MS/ DBB region. While paired patch-clamp recording is probably the only technique capable of showing unequivocally functional connectivity between two neurons, the technique alone is not adequate for quantification of connection ratios (Osten and Margrie 2013). We found little variability between EPSC and IPSC amplitudes among different cell types, indicating homogeneity of glutamatergic and GABAergic synapses within the MS/DBB. In addition, we showed reciprocity of connections between MS/DBB GABAergic and glutamatergic neurons. Cholinergic neurons also receive glutamatergic and GABAergic synapses emanating from other MS/DBB neurons. Taken together, our data demonstrate recurrence in MS/ DBB networks.
The existence of a recurrent network between septal neurons could synchronize MS/DBB neurons (Traub et al. 1996). Thus we assessed firing correlation between neurons belonging to different MS/DBB cell populations in the presence of kainate (Leao et al. 2012). While synchrony between APs from GABAergic neurons was higher than between APs from other combinations of neurons, overall, synchrony was relatively low among cell pairs. Cholinergic cells were the most rhythmic among the three populations studied, while glutamatergic neurons, as previously reported (Leao et al. 2012), display a very low firing frequency that precluded their inclusion in meaningful synchrony analysis. Series of spikes of cholinergic cells were also more rhythmic and predictable compared with series of spikes from the other neuronal classes when tested with RQA. Previously, it has been shown that GABAergic neurons in the MS/DBB fire bursts of APs in anesthetized and nonanesthetized rats (Simon et al. 2006). However, we found no evidence of burst firing in our recordings. This difference could arise from differences in reversal potentials due to ion concentration differences between our ACSF and the extracellular matrix, from severing of connections during slicing, and/or from a direct effect of kainate. In fact, we have previously shown that CA3 pyramidal cells show little bursting when bathed with kainate (Leao et al. 2009).

Some connectivity among MS/DBB neurons is preserved in $400-\mu \mathrm{m}$ coronal septal slices. but external inputs are practically lost. This loss reduces excitation, explaining the low firing rates of MS/DBB neurons observed in slice preparations (Garner et al. 2005). Firing rate changes are particularly striking in GABAergic neurons that dramatically decrease firing rates and lose bursting capabilities (Simon et al. 2006). In addition, slicing orientation, solutions, and subject age add up to the pool of variables that make network mapping in in vitro studies virtually impossible. In fact, there are studies demon-
A

A

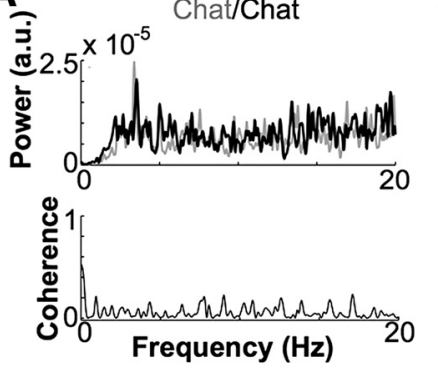

C
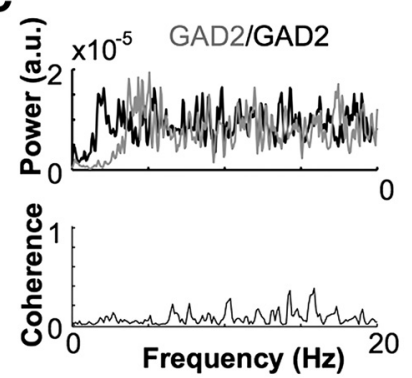

B
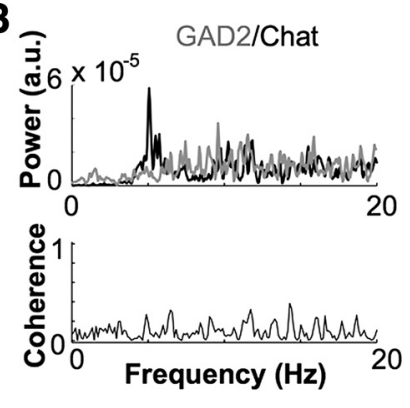

D

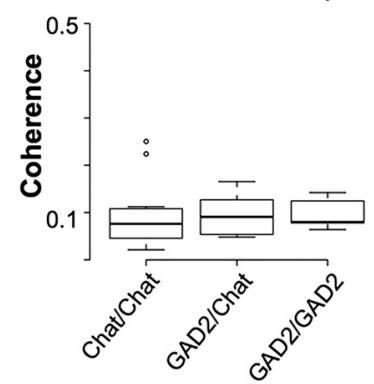

Fig. 4. Pairs of cholinergic neurons have higher spike coherence. $A-C$ : power spectral density (PSD) and squared coherence computed vs. frequency plot examples of spikes from ChAT/ChAT, GAD2/ChAT, and GAD2/GAD2 pairs, respectively. a.u., Arbitrary units. $D$ : box plot showing mean peak coherence from ChAT/ChAT, GAD2/ChAT, and GAD2/GAD2 neuron pairs. 

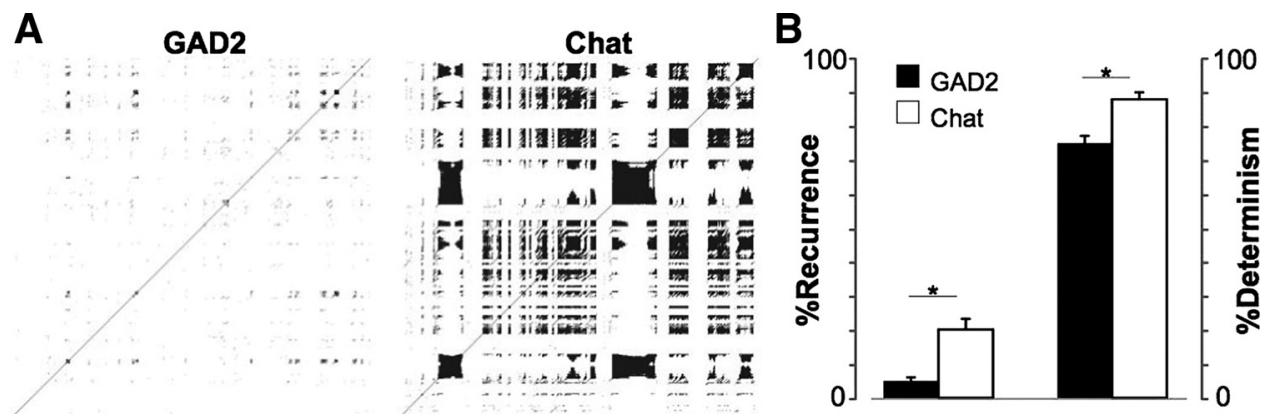

Fig. 5. Cholinergic neuron action potential firing shows higher predictability than firing in GAD2+ cells. A: recurrence plots of GAD2 + and ChAT + neurons. B: mean $\%$ recurrence and \% determinism (see MATERIALS AND METHODS for definitions) of GAD2 + and ChAT + MS/DBB neurons $\left({ }^{*} P<0.05, t\right.$-test $)$.

strating the existence of strong dipoles in sagittal MS/DBB slices capable of generating theta in the LFP in the presence of kainate (Garner et al. 2005). Nevertheless, circuits in in vitro preparations are crucial in determining the minimal circuits required for the generation of oscillatory activity (Börgers et al. 2012).

In the presence of kainate, cholinergic MS/DBB neurons fire regularly around $3 \mathrm{~Hz}$ with little synchrony in relation to spikes from other neurons. It has been shown that cholinergic neurons in vitro fire regularly in response to current injection or chemical stimulation (Unal et al. 2012; Wu et al. 2004). While a previous study has indicated that cholinergic neurons display little rhythmicity during theta (Simon et al. 2006), Vandecasteele et al. (2014) demonstrated that rhythmic stimulation of $\mathrm{ChAT}+$ neurons in the MS/DBB greatly enhances hippocampal theta. Thus it seems plausible that rhythmic activity from cholinergic neurons participates in theta generation.

Using synaptic data produced in this work and membrane properties of neurons derived from other studies (Leao et al.
2012; Unal et al. 2012; Wang 2002) we implemented a MS/ DBB network model to further investigate the role of intraseptal connectivity in the generation of regularity and synchronicity in spike activity. We found that increasing the number of intraseptal GABAergic or glutamatergic synapses or synaptic strength had little effect in producing regularity in GABAergic or glutamatergic neurons or interfering with intrinsic spiking regularity of cholinergic cells. Nevertheless, synchronicity was greatly enhanced when glutamatergic synapses were strengthened. Synchronicity through inhibition generally requires a high background firing rate (Traub et al. 1996), and glutamatergic neurons in the model (and in septal slices) fire sparsely (Leao et al. 2012). Interestingly, higher excitability of glutamatergic neurons (caused by lowering $I_{\mathrm{M}}$ ) in the model generated rhythmic spiking in glutamatergic and GABAergic neurons at gamma frequencies and disrupted low-frequency regular firing of cholinergic neurons. It has been proposed that faster rhythms (e.g., gamma) relate to the decay time constant
A

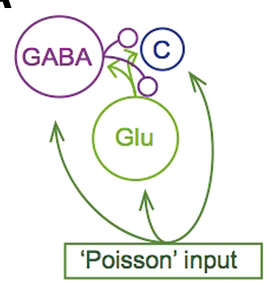

C

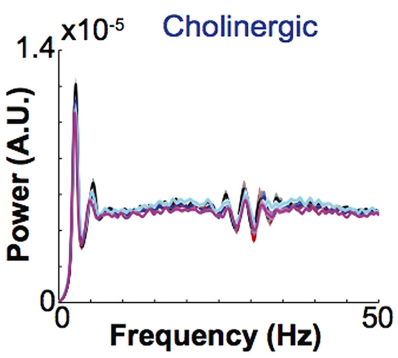

D

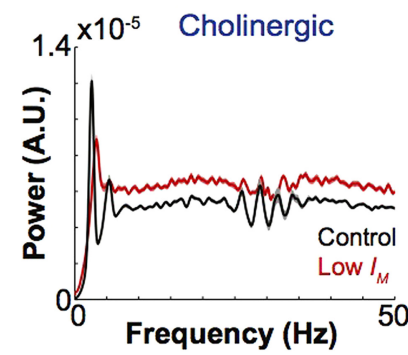

B

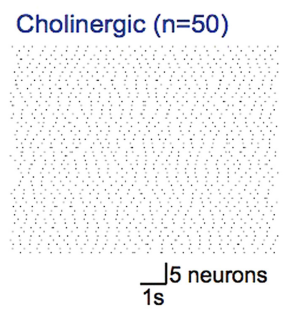

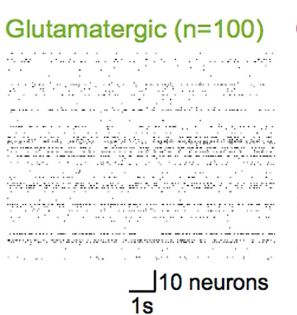

$1 \mathrm{~s}$
GABAergic $(n=100)$

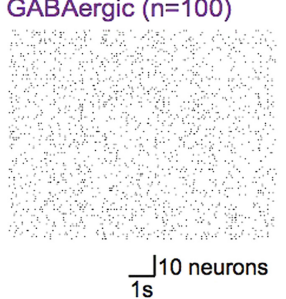

$\overrightarrow{1 s}$
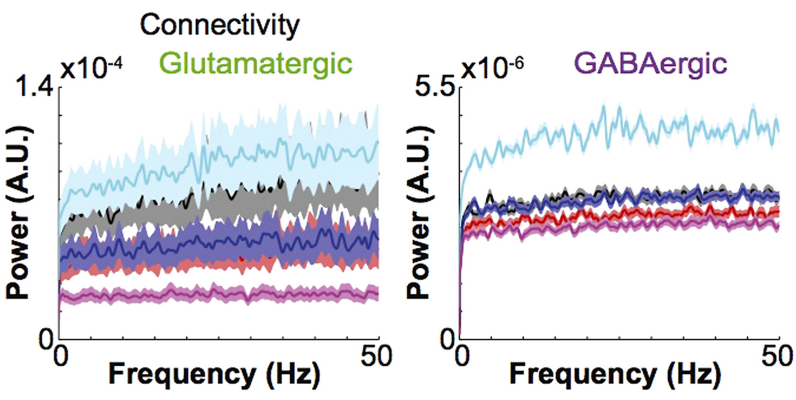

Low $I_{M}$ in glutamatergic neurons
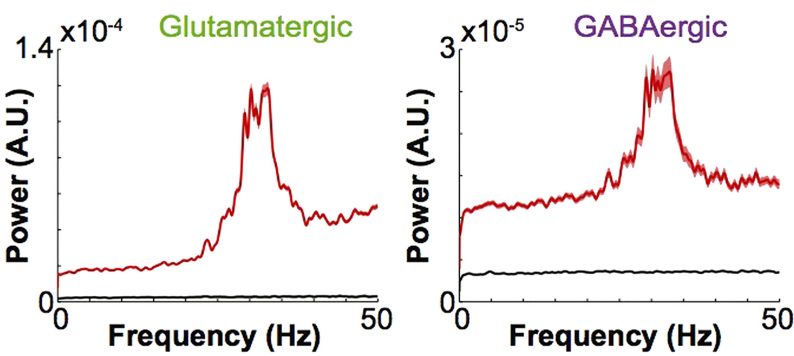

Fig. 6. Change in connectivity does not affect rhythmicity in MS/DBB model network. A: model network architecture. $B$ : raster plots showing cholinergic, glutamatergic, and GABAergic cells (control connectivity). $C$ : normalized PSD plots of different MS/DBB cell classes (black traces, control connectivity; red, $2 \times$ glutamatergic synapses; blue, $2 \times$ GABAergic synapses; cyan, $4 \times$ glutamatergic synapses; magenta, $4 \times$ GABAergic synapses; shaded areas represent \pm SE). $D$ : PSDs of cholinergic, glutamatergic, and GABAergic neurons in control conditions and when $g_{\mathrm{M}}$ (m-current conductance) in glutamatergic neurons was decreased from 1.8 to $0.7 \mathrm{nS}$. 
Table 2. Mean synchronization index among neuron pairs in MS/ DBB model with different connectivity

\begin{tabular}{lccc}
\hline \hline & Control & $4 \times$ GABAergic & $4 \times$ Glutamatergic \\
\hline Glu $\times$ Glu & 0.00059 & 0.00072 & 0.00054 \\
GABA $\times$ GABA & 0.00047 & 0.00130 & 0.00042 \\
Glu $\times$ GABA & 0.00043 & 0.05832 & 0.01287 \\
\hline
\end{tabular}

of the synaptic processes behind these oscillations (Faulkner et al. 1998; Traub et al. 1996). Thus it seems implausible that fast ionotropic GABAergic or glutamatergic synapses would underlie theta generation in a similar process as their involvement in gamma generation. Rather, voltage- and calcium-dependent ion channels (Hu et al. 2002) associated to recurrent GABAergic and glutamatergic connections could produce synchronous spiking of MS/DBB network. In addition, extraseptal afferents, includ-

ing feedback inputs from the hippocampus (Manseau et al. 2008; Stewart and Fox 1989), and/or ascending synchronizing inputs arising from the supramammillary and posterior hypothalamic nuclei (Colom 2006) could further organize the firing of MS/DBB neurons at theta frequencies.

In summary, our data support the notion that the interplay between the intrinsic membrane properties of the different MS/DBB neuronal cell classes with the recurrent connectivity among these cell classes underlies the ability of the MS/DBB to generate rhythmic network activity.

\section{GRANTS}

This work was supported by the Kjell och Märta Beijers Foundation; the Brazilian National Council for Scientific and Technological Development (R. N. Leão); National Institute of Neurological Disorders and Stroke Grant 5SC1 NS-065386 (L. V. Colom); and the Swedish Research Council (Veten-
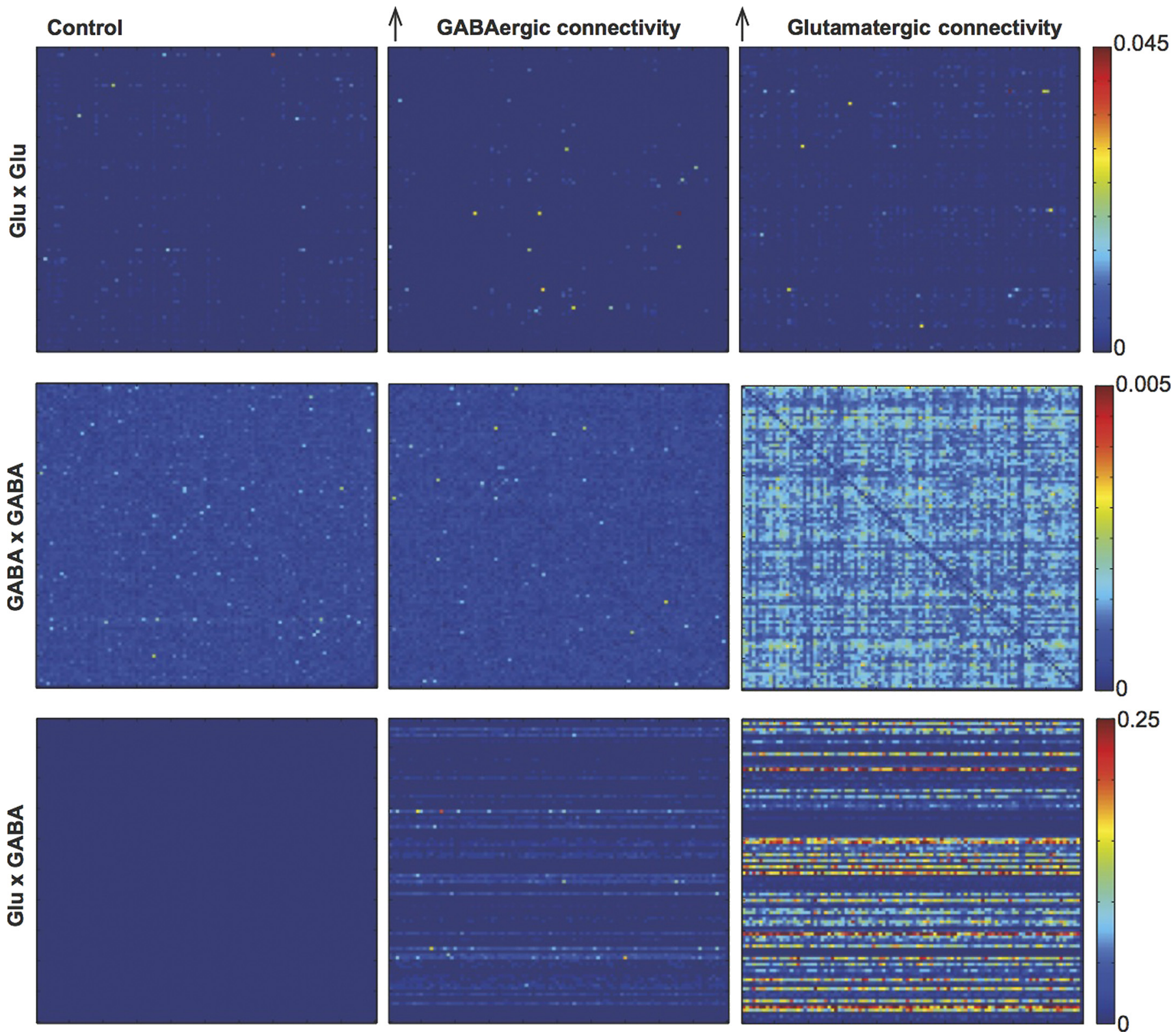

Fig. 7. Increasing the number of glutamatergic synapses increases synchrony. Synchronization index matrix (pairwise SI) of modeled glutamatergic and GABAergic neurons in different connection schemes (control and $4 \times$ more GABAergic and $4 \times$ more glutamatergic synapses). 
skapsrådet), the Swedish Medical Association (Svenska Läkaresällskapet), the Brain Foundation (Hjärnfonden), and the Strategic Program in Neurosciences at the Karolinska Institute (A. Fisahn).

\section{DISCLOSURES}

No conflicts of interest, financial or otherwise, are declared by the author(s).

\section{AUTHOR CONTRIBUTIONS}

Author contributions: R.N.L. and A.F. conception and design of research; R.N.L. and Z.H.T. performed experiments; R.N.L. and Z.H.T. analyzed data; R.N.L. interpreted results of experiments; R.N.L. prepared figures; R.N.L., L.V.C., and A.F. drafted manuscript; R.N.L., L.V.C., and A.F. edited and revised manuscript; R.N.L., Z.H.T., L.V.C., and A.F. approved final version of manuscript.

\section{REFERENCES}

Andersen P, Bland HB, Myhrer T, Schwartzkroin PA. Septo-hippocampal pathway necessary for dentate theta production. Brain Res 165: 13-22, 1979.

Bañuelos C, LaSarge CL, McQuail JA, Hartman JJ, Gilbert RJ, Ormerod BK, Bizon JL. Age-related changes in rostral basal forebrain cholinergic and GABAergic projection neurons: relationship with spatial impairment. Neurobiol Aging 34: 845-862, 2013.

Birgner C, Nordenankar K, Lundblad M, Mendez JA, Smith C, le Grevès M, Galter D, Olson L, Fredriksson A, Trudeau LE, Kullander K, Wallén-Mackenzie A. VGLUT2 in dopamine neurons is required for psychostimulant-induced behavioral activation. Proc Natl Acad Sci USA 107: 389-394, 2010.

Bland BH. The physiology and pharmacology of hippocampal formation theta rhythms. Prog Neurobiol 26: 1-54, 1986.

Bland BH, Colom LV. Extrinsic and intrinsic properties underlying oscillation and synchrony in limbic cortex. Prog Neurobiol 41: 157-208, 1993

Bland BH, Oddie SD, Colom LV. Mechanisms of neural synchrony in the septohippocampal pathways underlying hippocampal theta generation. $J$ Neurosci 19: 3223-3237, 1999.

Börgers C, Talei Franzesi G, Lebeau FE, Boyden ES, Kopell NJ. Minimal size of cell assemblies coordinated by gamma oscillations. PLoS Comput Biol 8: e1002362, 2012.

Borgius L, Restrepo CE, Leao RN, Saleh N, Kiehn O. A transgenic mouse line for molecular genetic analysis of excitatory glutamatergic neurons. Mol Cell Neurosci 45: 245-257, 2010.

Buzsáki G. Theta oscillations in the hippocampus. Neuron 33: 325-340, 2002.

Colom LV. Septal networks: relevance to theta rhythm, epilepsy and Alzheimer's disease. J Neurochem 96: 609-624, 2006.

Colom LV, Castaneda MT, Reyna T, Hernandez S, Garrido-Sanabria E. Characterization of medial septal glutamatergic neurons and their projection to the hippocampus. Synapse 58: 151-164, 2005.

Diamond DM, Dunwiddie TV, Rose GM. Characteristics of hippocampal primed burst potentiation in vitro and in the awake rat. $J$ Neurosci 8: 4079-4088, 1988

Faulkner HJ, Traub RD, Whittington MA. Disruption of synchronous gamma oscillations in the rat hippocampal slice: a common mechanism of anaesthetic drug action. Br J Pharmacol 125: 483-492, 1998.

Garner HL, Whittington MA, Henderson Z. Induction by kainate of theta frequency rhythmic activity in the rat medial septum-diagonal band complex in vitro. J Physiol 564: 83-102, 2005.

Garrido-Sanabria ER, Perez-Cordova MG, Colom LV. Differential expression of voltage-gated $\mathrm{K}^{+}$currents in medial septum/diagonal band complex neurons exhibiting distinct firing phenotypes. Neurosci Res 70: 361-369, 2011.

Gaspar P, Berger B, Lesur A, Borsotti JP, Febvret A. Somatostatin 28 and neuropeptide $\mathrm{Y}$ innervation in the septal area and related cortical and subcortical structures of the human brain. Distribution, relationships and evidence for differential coexistence. Neuroscience 22: 49-73, 1987.

Glasgow SD, Chapman CA. Conductances mediating intrinsic theta-frequency membrane potential oscillations in layer II parasubicular neurons. $J$ Neurophysiol 100: 2746-2756, 2008.

Goodman D, Brette R. Brian: a simulator for spiking neural networks in Python. Front Neuroinformatics 2: 5, 2008.
Henderson Z, Lu CB, Janzsó G, Matto N, McKinley CE, Yanagawa Y, Halasy K. Distribution and role of Kv3.1b in neurons in the medial septum diagonal band complex. Neuroscience 166: 952-969, 2010.

Hilscher MM, Leão KE, Leão RN. Synchronization through nonreciprocal connections in a hybrid hippocampus microcircuit. Front Neural Circuits 7: $120,2013$.

Hodgkin AL, Huxley AF. A quantitative description of membrane current and its application to conduction and excitation in nerve. J Physiol 117: 500544, 1952.

Hu H, Vervaeke K, Storm JF. Two forms of electrical resonance at theta frequencies, generated by $\mathrm{M}$-current, h-current and persistent $\mathrm{Na}^{+}$current in rat hippocampal pyramidal cells. J Physiol 545: 783-805, 2002.

Huh CY, Goutagny R, Williams S. Glutamatergic neurons of the mouse medial septum and diagonal band of Broca synaptically drive hippocampal pyramidal cells: relevance for hippocampal theta rhythm. J Neurosci 30: 15951-15961, 2010.

Hung CP, Ramsden BM, Roe AW. Weakly modulated spike trains: significance, precision, and correction for sample size. J Neurophysiol 87: 25422554, 2002.

Jensen O, Lisman JE. Position reconstruction from an ensemble of hippocampal place cells: contribution of theta phase coding. J Neurophysiol 83: 2602-2609, 2000.

Kiss J, Csáki A, Bokor H, Shanabrough M, Leranth C. The supramammillo-hippocampal and supramammillo-septal glutamatergic/aspartatergic projections in the rat: a combined $\left[{ }^{3} \mathrm{H}\right] \mathrm{D}$-aspartate autoradiographic and immunohistochemical study. Neuroscience 97: 657-669, 2000.

Kocsis B, Li S. In vivo contribution of h-channels in the septal pacemaker to theta rhythm generation. Eur J Neurosci 20: 2149-2158, 2004.

Leao RN, Colom LV, Borgius L, Kiehn O, Fisahn A. Medial septal dysfunction by $\mathrm{A} \beta$-induced KCNQ channel-block in glutamatergic neurons. Neurobiol Aging 33: 2046-2061, 2012.

Leao RN, Leao FN, Walmsley B. Non-random nature of spontaneous mIPSCs in mouse auditory brainstem neurons revealed by recurrence quantification analysis. Proc Biol Sci 272: 2551-2559, 2005.

Leao RN, Tan HM, Fisahn A. Kv7/KCNQ channels control action potential phasing of pyramidal neurons during hippocampal gamma oscillations in vitro. J Neurosci 29: 13353-13364, 2009.

Manseau F, Goutagny R, Danik M, Williams S. The hippocamposeptal pathway generates rhythmic firing of GABAergic neurons in the medial septum and diagonal bands: an investigation using a complete septohippocampal preparation in vitro. J Neurosci 28: 4096-4107, 2008.

Marwan N, Wessel N, Meyerfeldt U, Schirdewan A, Kurths J. Recurrenceplot-based measures of complexity and their application to heart-ratevariability data. Phys Rev E Stat Nonlin Soft Matter Phys 66: 026702, 2002.

Mehta MR. Role of rhythms in facilitating short-term memory. Neuron 45: 7-9, 2005.

Mitchell SJ, Rawlins JN, Steward O, Olton DS. Medial septal area lesions disrupt theta rhythm and cholinergic staining in medial cortex and produce impaired radial arm maze behavior in rats. J Neurosci 2: 292-302, 1982.

Morris NP, Fyffe RE, Robertson B. Characterisation of hyperpolarizationactivated currents $\left(I_{\mathrm{h}}\right)$ in the medial septum/diagonal band complex in the mouse. Brain Res 1006: 74-86, 2004.

Osten P, Margrie TW. Mapping brain circuitry with a light microscope. Nat Methods 10: 515-523, 2013.

Simon AP, Poindessous-Jazat F, Dutar P, Epelbaum J, Bassant MH. Firing properties of anatomically identified neurons in the medial septum of anesthetized and unanesthetized restrained rats. J Neurosci 26: 9038-9046, 2006.

Sotty F, Danik M, Manseau F, Laplante F, Quirion R, Williams S. Distinct electrophysiological properties of glutamatergic, cholinergic and GABAergic rat septohippocampal neurons: novel implications for hippocampal rhythmicity. J Physiol 551: 927-943, 2003.

Stewart M, Fox SE. Firing relations of medial septal neurons to the hippocampal theta rhythm in urethane anesthetized rats. Exp Brain Res 77: 507-516, 1989.

Teles-Grilo Ruivo LM, Mellor JR. Cholinergic modulation of hippocampal network function. Front Synaptic Neurosci 5: 2, 2013.

Traub RD, Whittington MA, Colling SB, Buzsáki G, Jefferys JG. Analysis of gamma rhythms in the rat hippocampus in vitro and in vivo. $J$ Physiol 493: 471-484, 1996.

Unal CT, Golowasch JP, Zaborszky L. Adult mouse basal forebrain harbors two distinct cholinergic populations defined by their electrophysiology. Front Behav Neurosci 6: 21, 2012.

Vandecasteele M, Varga V, Berenyi A, Papp E, Bartho P, Venance L, Freund TF, Buzsaki G. Optogenetic activation of septal cholinergic neu- 
rons suppresses sharp wave ripples and enhances theta oscillations in the hippocampus. Proc Natl Acad Sci USA 111: 13535-13540, 2014.

Varga V, Hangya B, Kránitz K, Ludányi A, Zemankovics R, Katona I, Shigemoto R, Freund TF, Borhegyi Z. The presence of pacemaker HCN channels identifies theta rhythmic GABAergic neurons in the medial septum. J Physiol 586: 3893-3915, 2008.

Vinogradova OS. Expression, control, and probable functional significance of the neuronal theta-rhythm. Prog Neurobiol 45: 523-583, 1995.
Wang XJ. Pacemaker neurons for the theta rhythm and their synchronization in the septohippocampal reciprocal loop. J Neurophysiol 87: 889-900, 2002.

Wu M, Hajszan T, Xu C, Leranth C, Alreja M. Group I metabotropic glutamate receptor activation produces a direct excitation of identified septohippocampal cholinergic neurons. J Neurophysiol 92: 1216-1225, 2004.

Zbilut JP, Webber CL. Embeddings and delays as derived from quantification of recurrence plots. Phys Lett A 171: 199-203, 1992.

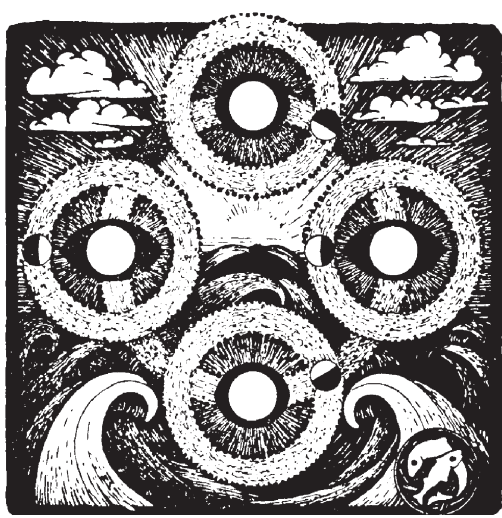

